\title{
Erratum to: Abstracts of the 53rd Annual Meeting of the EASD, Lisbon 2017. Abstract 788: 'Pharmacokinetics and tolerability of oral semaglutide in subjects with renal impairment'
}

T. W. Anderson ${ }^{1}$ - C. Granhall ${ }^{1}$ - Á. Réthy ${ }^{2}$ - F. L. Søndergaard ${ }^{1} \cdot$ M. Thomsen ${ }^{1}$

(C) Springer-Verlag GmbH Germany 2017

Erratum to: Diabetologia

http://dx.doi.org/10.1007/s00125-017-4350-Z

T.W. Anderson ${ }^{1}$, C. Granhall ${ }^{1}$, Á. Réthy ${ }^{2}$, F.L. Søndergaard ${ }^{1}$, M. Thomsen ${ }^{1}$

${ }^{1}$ Novo Nordisk, Søborg, Denmark, ${ }^{2}$ Péterfy Sándor utcai Kórház, Budapest, Hungary.

The original version of this abstract unfortunately contained a mistake. Instead of a table a figure was erroneously published. The correct table is given below.

Semaglutide exposure in subjects with renal impairment versus subjects with normal renal function

\begin{tabular}{lcc}
\hline & $\begin{array}{c}\text { AUC } \text { 0-24h,Day 10 } \\
\text { Ratio [90\% Cl] }\end{array}$ & $\begin{array}{c}\mathrm{C}_{\text {max,Day 10 }} \\
\text { Ratio [90\% Cl] }\end{array}$ \\
\hline Mild vs. Normal & $1.37[0.91 ; 2.06]$ & $1.39[0.93 ; 2.06]$ \\
Moderate vs. Normal & $1.13[0.69 ; 1.84]$ & $1.20[0.75 ; 1.93]$ \\
Severe vs. Normal & $0.61[0.42 ; 0.88]$ & $0.61[0.42 ; 0.87]$ \\
ESRD vs. Normal & $1.02[0.59 ; 1.79]$ & $1.06[0.61 ; 1.84]$ \\
\hline
\end{tabular}

AUC 0 -24h,Day 10: Area under the semaglutide plasma concentration-time curve from 0 to 24 hours after the $10^{\text {th }}$ daily dose

$\mathrm{C}_{\text {max }, \text { Day 10: }}$ Maximum semaglutide plasma concentration after the $10^{\text {th }}$ daily dose

The online version of the original article can be found at http://dx.doi.org/ $10.1007 / \mathrm{s} 00125-017-4350-\mathrm{z}$

T. W. Anderson

1 Novo Nordisk, Søborg, Denmark

2 Péterfy Sándor utcai Kórház, Budapest, Hungary 